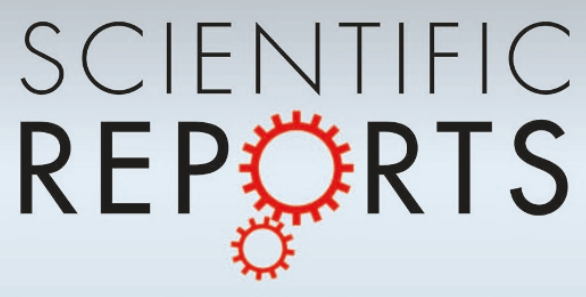

OPEN

SUBJECT AREAS:

SUPERCONDUCTING

PROPERTIES AND

MATERIALS

STRUCTURAL PROPERTIES

PHASE TRANSITIONS AND CRITICAL PHENOMENA

Received

23 October 2014

Accepted

11 December 2014

Published

16 January 2015

Correspondence and requests for materials should be addressed to V.M.V. (vinokour@anl.

\section{Rayleigh approximation to ground state of the Bose and Coulomb glasses}

\author{
S. D. Ryan', V. Mityushev², V. M. Vinokur ${ }^{3} \&$ L. Berlyand'
}

'Department of Mathematics, The Pennsylvania State University, University Park, PA 16802, USA, ${ }^{2}$ Department of Computer
Sciences and Computer Methods, Pedagogical University, ul.Podchorazych 2, Krakow 30-084, Poland, ${ }^{3}$ Materials Science
Division, Argonne National Laboratory, 9700 S. Cass Ave, Argonne, Illinois 60439, USA.

Glasses are rigid systems in which competing interactions prevent simultaneous minimization of local energies. This leads to frustration and highly degenerate ground states the nature and properties of which are still far from being thoroughly understood. We report an analytical approach based on the method of functional equations that allows us to construct the Rayleigh approximation to the ground state of a two-dimensional (2D) random Coulomb system with logarithmic interactions. We realize a model for 2D Coulomb glass as a cylindrical type II superconductor containing randomly located columnar defects (CD) which trap superconducting vortices induced by applied magnetic field. Our findings break ground for analytical studies of glassy systems, marking an important step towards understanding their properties.

U nderstanding the complexity of glassy behaviors resulting from the exponential degeneracy of their ground states is key to gaining insight into a variety of systems in nature, including spins and magnetic moments in magnetism ${ }^{1-3}$, electrons in metals ${ }^{4,5}$, Cooper pairs in disordered superconductors ${ }^{6,7}$, vortices and interfaces in superconductors and magnets ${ }^{1,8-11}$, social ${ }^{12}$ and neural ${ }^{13}$ networks, and protein folding ${ }^{14}$ remains $^{2}$ one of the major challenges in physics. One of the furthest-reaching problems in the physics of glasses is that of understanding the nature of Coulomb or electronic glasses where concurring effects of long-range Coulomb interactions and disorder result in a depletion of the density of electronic states (referred to as the opening of a 'Coulomb gap') which turns metal into an insulator. This causes two key aspects of glassiness, namely disorder and frustration, to become maximally pronounced. Disorder means that, unlike periodic structures, glasses do not possess long-range order, while frustration refers to the competition among conflicting interactions. Consequently, the system does not find an accommodation that complies with all constraints but arrives instead at a multitude of degenerate, i.e. almost equally advantageous states the number of which exponentially grows with the system size. These states are separated by nearly infinitely large free-energy barriers in a phase space that acquires complex hierarchical structure. As a result, glasses are effectively non-ergodic and manifestly lack equilibrium, that is they cannot equilibrate with their environment. This gives rise to remarkable aging and memory effects and highly nonlinear dynamics. The reason that the properties of glasses are still not thoroughly understood is that the same complex structure of a highly degenerate ground state that makes glasses interesting and appealing, impedes the application of our standard theoretical physics machinery. Moreover, frustration and non-ergodicity denigrate efficiency of even the best numerical algorithms, which are commonly used to study glasses, because there is no guarantee that the process of a search for the ground state will take the system close to the global minimum rather than getting stuck within some local potential well, see refs 15-17 and references therein. The nature of the ground state of Coulomb or electronic glasses poses a special challenge since electronic glasses are agreeably at the heart of physics of many strongly correlated systems, most notably, high temperature superconductors ${ }^{18}$.

Here we address this challenge and offer an analytical approach to construct an approximation to a ground state of the 2D random finite system in the first order with respect to disorder. We consider a two-dimensional Coulomb gas subject to quenched disorder and take advantage of the latter's equivalence to the system of 3D vortices in type II superconductors containing randomly distributed columnar defects ${ }^{9}$, see Fig. 1a. Complete identity is achieved by choosing a lateral size for the system not exceeding the London penetration depth, $\lambda$. Then vortex-vortex logarithmic interactions remain unscreened, and the vortex system becomes isomorphic to the $2 \mathrm{D}$ Coulomb gas of logarithmically interacting electric charges, Fig. 1b, c. Hence the equivalence of the problems of the lowest energy states of these two systems: the global minimum of the Ginzburg-Landau (GL) functional, describing the configuration of vortices corresponding to the lowest energy, defines the ground state of both. 

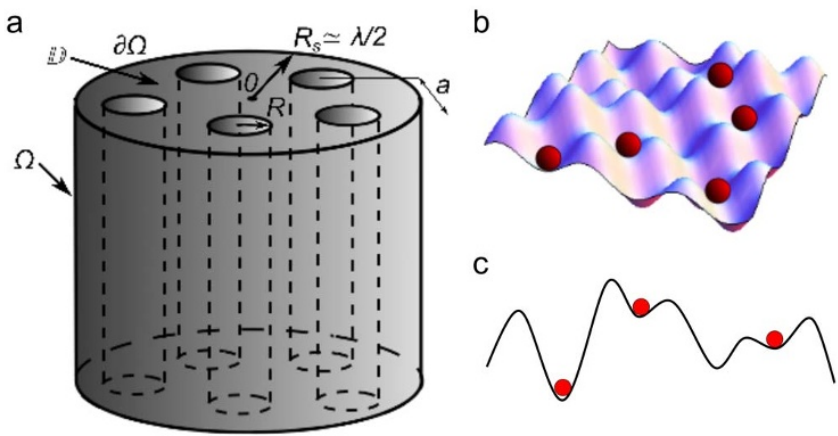

C

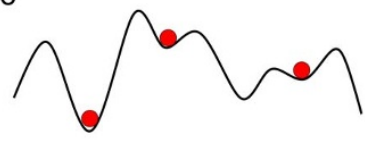

Figure 1 Superconducting cylinder with columnar defects and corresponding single-particle potential relief. (a): Sketch of a superconducting cylinder with arbitrarily distributed columnar defects. The characteristic lengths are related by the inequalities chain, $\xi \ll R \ll a \ll R_{\mathrm{s}} \simeq \lambda$. (b): Egg-crate energy potential relief for a single particle in the related two-dimensional Coulomb gas of charged particles. (c): Cross-section of the egg-crate potential relief for a charge emphasizing its random character.

We consider a large, i.e. containing a macroscopic number of defects, but finite superconducting sample in a form of a cylinder, with a circular base of the diameter $R_{\mathrm{s}} \simeq \lambda / 2$. The cylinder is stuffed with columnar cavities or defects of radius $R$, mean spacing between defects being $a$, see Fig. 1a. We take $\kappa \equiv \lambda / \xi \gg 1$, and assume the chain of inequalities, $\xi \ll R \ll a \ll \lambda$, where $\xi$ is the superconducting coherence length. In addition we impose the even stronger scale separation condition that $|\log (\kappa)| \gg|\log (R / \lambda)|$. We restrict ourselves to the range of magnetic fields $H<H_{\mathrm{c} 1}$, where $H_{\mathrm{c} 1}$ is the lower critical field, so that vortices do not exist in the bulk of the sample but can only be trapped by CDs. We develop an analytical approach for finding the vortex distribution $\left\{d_{j}\right\}, j=1,2, \cdots, N$, where vorticity or degree $\left\{d_{j}\right\}$ measures the number of flux quanta trapped by the $j$ th $\mathrm{CD}$, minimizing the system energy, i.e. for finding the vortex distribution corresponding to the ground state of the system. The maximal filling of a CD is defined by the condition ${ }^{19} n_{\max }=[R / 2 \xi]$. Vortex quantization, i.e. the condition that the degrees must be integers imposes a constraint on the minimization of the energy. Our technique is based on the method of functional equations ${ }^{20-24}$, see Methods and Supplementary Information (SI) for details. That the found energy-minimizing distribution (minimizer) corresponds to a unique global energy minimum follows from convexity of the energy functional. If the array of CDs is regular, the vortex system can assume a terrace-like ground state i.e. form a hierarchical nested domain structure ${ }^{25,26}$.

Each domain is characterized by its filling factor $\left\{d_{j}\right\}$, which grows upon traversing from the sample border to its center. To emphasize the net effects of disorder we consider initially a regular arrangement of CDs and then watch how the nested domain's ground state structure evolves upon randomizing the distribution of the defects. We reveal that, as the randomness grows, the vortex count within each vortex domain remains approximately the same but the smooth domain walls of the regular defect array acquire fractal character and turn into fractal interfaces with non-universal fractal dimensionality depending on the degree of disorder. We find that disorder generates energy states for vortices, that lie below those corresponding to the regular array of CDs. These low-lying vortex energy states that can be viewed as a hallmark of emergent glassiness manifest themselves, in particular, in decreasing the field of the first vortex trapping with increasing degree of disorder.

We describe our system with the Ginzburg-Landau (GL) functional:
$\mathcal{F}=$
$\int_{\Omega}\left[\frac{\hbar}{4 m}\left|\left(\nabla-\frac{2 i e}{\hbar c} \mathbf{A}\right) \Psi\right|^{2}+\alpha|\Psi|^{2}+\frac{\beta}{2}|\Psi|^{4}+\frac{1}{8 \pi}(\operatorname{curl} \mathbf{A}-\mathbf{H})^{2}\right] d^{2} \mathbf{r} d z$,

where $\Omega$ is the volume of the sample, $\Psi(\mathbf{r})$ is the superconducting order parameter, $\mathrm{H}$ is the external magnetic field applied along the $z$ axis aligned with the cylinder, $\mathbf{r}$ the coordinate vector in the plane of $\Omega$ perpendicular to $z, m$ and $-e$ are the electron mass and charge, respectively, and $\mathrm{A}(\mathbf{r})$ is the vector potential of the magnetic field so that $\mathbf{B}=\operatorname{curl}(\mathbf{A})$. The London penetration depth $\lambda^{2}=\left(m c^{2} \beta\right) /$ $\left(8 \pi e^{2}|\alpha|\right)$ and the coherence length $\xi^{2}=\hbar^{2} /(4 m|\alpha|)$ are encoded in the coefficients of the GL functional. For the sake of convenience, we define the standard dimensionless order parameter $u=\Psi / \Psi_{0}$ where $\Psi_{0}=-\sqrt{\alpha / \beta}$, measure lengths in units of $\lambda$ and the magnetic field in the units of $\Phi_{0} /(2 \pi \lambda \xi), \Phi_{0}=\pi \hbar c / e$ is the magnetic flux quantum. The dimensionless columnar defect radius $\rho=R / \lambda \ll \varepsilon \equiv a / \lambda \ll R_{\mathrm{s}}$ $\equiv(1 / 2) \operatorname{diam}(\Omega) / \lambda \approx 1$. We consider the case when the CD's radii are small as compared to their spacing and are parameterized by $\rho=$ $\exp \left(-\gamma / \varepsilon^{2}\right)$, where $\gamma$ is a constant of the order of unity, which implies an exponential separation of the characteristic lengths. Since the spatial scale of the variation of the order parameter $\xi$ is the smallest scale in the problem, we can set the order parameter $|u|=1$ everywhere besides the CDs. It has been established ${ }^{25,26}$ that the distribution of vortices obtained from the minimization of (1) is identical to that obtained by minimizing the energy

$$
E(h)=\frac{1}{2} \int_{\Omega}|\nabla h|^{2} d^{2} \mathbf{r} d z+\frac{1}{2} \int_{\Omega}\left(h-h_{\mathrm{a}}\right)^{2} d^{2} \mathbf{r} d z,
$$

where the distribution of the field corresponding to the ground state of the system, or the minimizer $h=h\left(\left\{d_{k}\right\}\right)$ satisfies the following Euler-Lagrange equations

$$
-\Delta h+h=2 \pi \mu(\mathbf{r})
$$

with $\mu(\mathbf{r})=\sum_{j} d_{\mathrm{j}} \delta\left(\mathbf{r}-\mathbf{r}_{\mathrm{j}}\right)$ ensuring the correct boundary conditions at the CDs interfaces: $h(\mathbf{r})=h_{\mathrm{a}}=$ constant on the exterior boundary and constant boundary conditions on interfaces, $d_{j}$ represents the degree of the vortex in the $j$ th $\mathrm{CD}$, i.e. the number of flux quanta trapped by $j$ th $C D$. The task now is to derive the unique global minimizer of (2) as an asymptotic solution to boundary value problem (3) for the magnetic field given an $n$-tuple of degrees $\mathbf{d}=\left(d_{1}, \ldots\right.$, $d_{\mathrm{N}}$ ), $N$ being the total number of CDs. To this end one first finds the magnetic field $h$ as a function of the degrees by employing the analytical method of functional equations developed for Poisson equa$\operatorname{tion}^{23}$, which we outline below, see also SI. The derived $h\left(\left\{d_{j}\right\}\right)$ is plugged in into the energy functional (2), and then the numerical minimization over the class of all $n$-tuples of degrees with the additional constraint that each degree must be an integer is carried out.

We seek an analytical solution to (3) as a series $h=h_{\mathrm{a}}\left(h^{(0)}+h^{(1)}+\right.$ $\left.h^{(2)}+\ldots\right)$ with respect to a small parameter $1 /|\ln \rho|$, where $h^{(0)}=\frac{I_{0}(|\mathbf{r}|)}{I_{0}(1)}, I_{0}(x)$ is the modied Bessel function of order zero and each term $h^{(k)}=O\left(1 /\left|\ln ^{k} \rho\right|\right)$. This expansion solves equations from the cascade of problems ${ }^{27}: \Delta h^{(0)}=h^{(0)}, \Delta h^{(1)}=0, \Delta h^{(2)}=h^{(1)}, \ldots, \Delta h^{(k)}$ $=h^{(k-1)}$. We find the minimizer for the random distribution of CDs within Rayleigh approximation truncating the expansion at the lowest order terms ${ }^{28,29}$. Let $\zeta=x_{1}+i x_{2}$ be a complex variable corresponding to $\mathbf{r}$ and complex numbers $a_{\mathrm{k}}$ be coordinates of CD centers 
in the $\zeta$-plane. We look for a solution to $(3)$ in the form $h \approx h_{\mathrm{a}}\left(h^{(0)}+\right.$ $\left.h^{(1)}\right)$ with

$$
h^{(1)}(\zeta)=\operatorname{Re} \varphi(\zeta), \quad \zeta \in D
$$

where the perforated domain $D$ is a cross section of $\Omega$ and $\varphi(\zeta)$ is an unknown function holomorphic in $D$. One can prove the convergence of the successive approximations for the functional equations for $\psi(\zeta)$ (see SI), where $\psi(\zeta)=\varphi^{\prime}(\zeta)$. The solution to (4) in the multiconnected domain $D$ is found up to the order of $O\left(1 / \ln ^{2} \rho\right)$ as

$\varphi(\zeta)=c_{0}+\sum_{m=1}^{\mathrm{N}} \beta_{\mathrm{m}}\left[\ln \left(\zeta-a_{\mathrm{m}}\right)-\ln \left(\frac{1}{\bar{a}_{\mathrm{m}}}-\zeta\right)\right]+O\left(\rho^{2}\right), \quad \zeta \in D$,

with unknown $c_{0} \in \mathbb{R}$. From the boundary conditions at the CDs, we express $c_{0}$ linearly through $\beta_{k}$ and derive a system of linear algebraic equations for the constants $\beta_{k}, k=1, \ldots, N$. The coefficients of this system depend on $\rho$ and we retain terms up to $O\left(1 / \ln ^{2} \rho\right)$. This system has a dominant diagonal part for small $\rho$ implying the uniqueness of the solution. One can observe that in the lowest order in $1 / \ln \rho$ terms, $\beta_{k} \sim 1 / \ln \rho$ and hence $h^{(1)} \sim 1 / \ln \rho$. If more terms are included into the Rayleigh expansion (with each term $h^{(p)} \sim 1 / \ln ^{p} \rho$ ), then the series $\sum_{p=0}^{\infty} h^{(p)}$ would converge to $h$. The linear system for $\beta_{k}$ can be solved numerically which completes the calculation of the magnetic field $h$ in the Rayleigh approximation for given $\mathbf{d}=\left(d_{1}, \ldots\right.$, $d_{\mathrm{N}}$ ). Once all $\beta_{k}$ are obtained, we minimize the energy as a function of degrees, $\mathbf{d}$, and determine $\mathbf{d}$ corresponding to the energy minimum. Since the energy functional is convex, the local minimizer is a global minimizer, which solves the problem of finding the ground state. The derivation of $\mathbf{d}=\left(d_{1}, \ldots, d_{\mathrm{N}}\right)$ for random CD array constitutes the main result of our work.

A fundamental implication of the randomization of the CD arrangement is the formation of a Bose glass state endowed with arbitrarily low-lying energy states for vortices ${ }^{9}$. While the Rayleigh approximation cannot guarantee the construction of a true glassy state, the observed decrease of the effective lower critical field, i.e. the field of the first vortex trapping $H_{\mathrm{G}}$ as compared to the critical field $H_{\text {reg }}$ where first vortices appear at the regular (periodic) $\mathrm{CD}$ array, can be viewed as a direct manifestation of emerging glassiness in a finite superconducting cylinder. Shown in Fig. 2a, b is the is the behavior of $H_{\mathrm{G}}$ as function of the degree of disorder and of the total number of CDs in a fully randomized array. Trapping vortices modifies the phase diagram of the type II superconductor as shown in Fig. 2c.

The emergence of glassy features in the sample containing $N=$ 770 columnar defects and $n_{\mathrm{v}}=1105$ vortices is illustrated in Fig. 3 . Initially CDs are arranged into a regular lattice. Accordingly, vortices form two nested domains with the degrees $d=0$ and $d=1$. Panels ac of Fig. 3 demonstrates how the terrace structure of the nested domains evolves and gets blurred upon incremental growth of the randomness in the CD's locations. While the domains corresponding to different degrees retain their identities, the interfaces between them acquire a fractal structure. Making use of the Box-Counting $\operatorname{method}^{30}$, we find the fractal dimension of the interface, $\mathcal{D}:=-\ln \left(N_{a}\right) / \ln \left(s_{a}\right)$, where $N_{a}$ is the number of cells the interface curve crosses and $s_{a}$ is the scale for the trial $a$. The evolution of the interface between the $d=0$ and $d=1$ domains, clearly seen in Fig 3, visualizes incremental fractalization. The fractal dimensionality as a function of the degree of disorder quantified by the number of steps $M$ in the randomization procedure, see SI, is presented in Fig. 3d. Its initial part is reasonably fitted as $\mathcal{D}(M)=c M^{v}+1.000$ with $c=0.060$ \pm 0.01 and $v=0.335 \pm 0.01$. Upon further increase of the random steps, fractal dimensionality converges to $\mathcal{D}=1.286 \pm 0.021$.
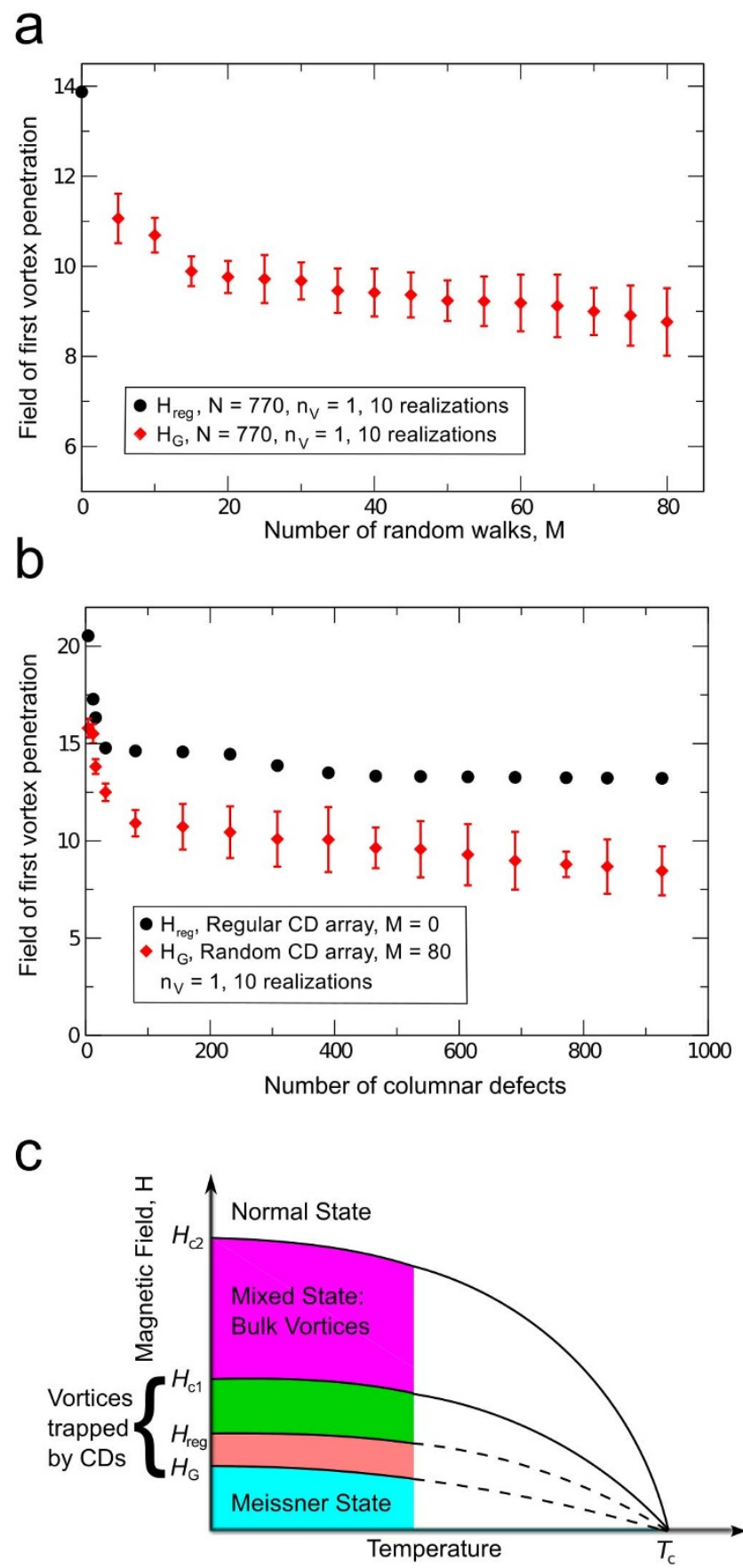

Figure $2 \mid$ Emergence of glass. (a): The field of the first emergence of a vortex trapped by $\mathrm{CD}, H_{\mathrm{G}}$, as function of the number of the randomization steps $M$. Incremental decrease of $H_{\mathrm{G}}$ reflects the decrease of the lowest energy of the vortex trapped by CD, i.e. emergence of glassiness. (b): Fields of the first penetration $H_{\text {reg }}$ for periodic array of CDs and $H_{\mathrm{G}}$ for random array as functions of the number of CDs in the sample. Upon increasing the number of CDs, $H_{\text {reg }}$ saturates, but $H_{\mathrm{G}}$ continues to gradually decrease. This illustrates the increasing glassiness of the system: as the number of CDs grows, more and more deep potential wells associated with CDs appear. (c): Low-temperature part of the $H$-T vortex phase diagram.

Fractalization of the interface leads to the shrinking of the vortexfree shell near the sample surface: its average width first decreases with the growing level of randomness (see Fig. 3e) as $c_{1} M^{v_{1}}+c_{2}$, with $v_{1}=0.323 \pm 0.01, c_{1}=-0.025 \pm 0.01$, and $c_{2}=0.257 \pm 0.01$, and then saturates, which parallels the behavior of $\mathcal{D}(M)$. Note that $v_{1}$ appears to be close to $v$. 

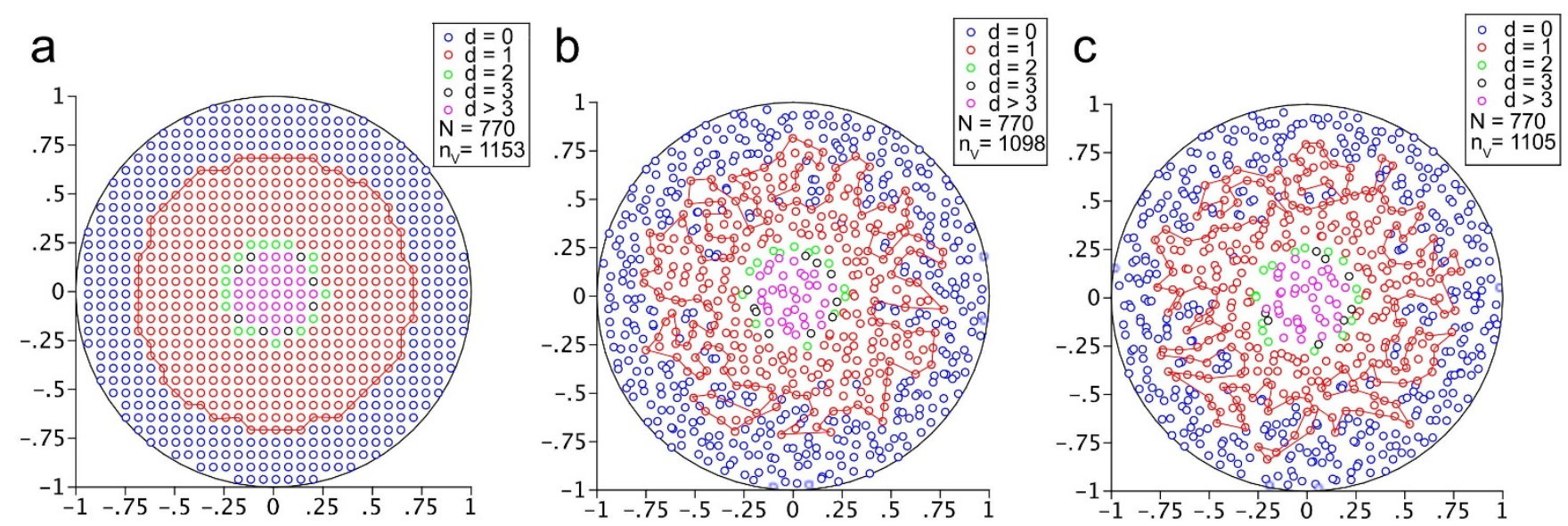

d

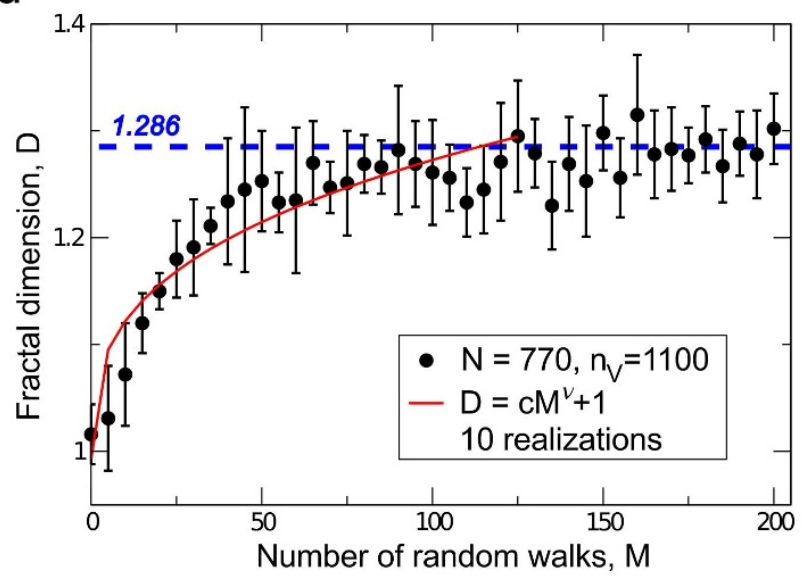

e

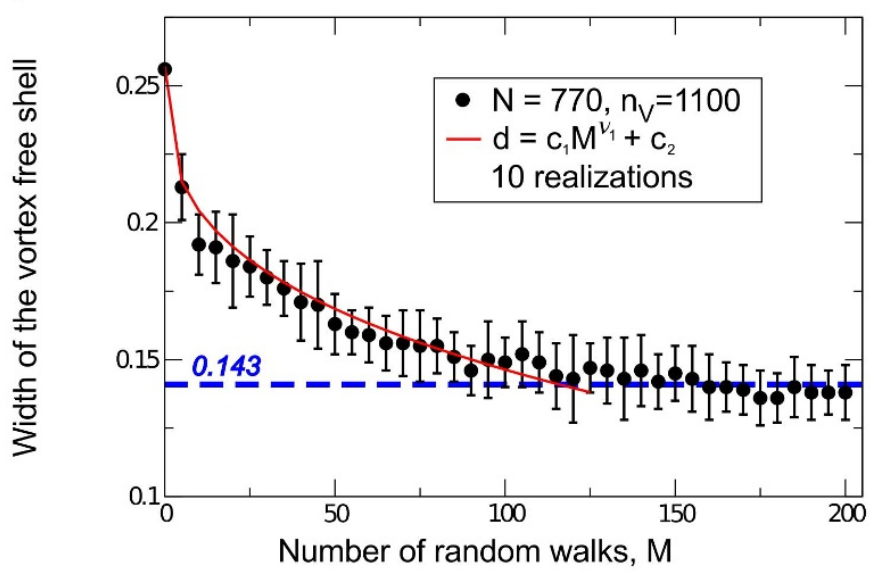

Figure 3 Fractal dimension of vortex domain interface. (a)-(c): Developing fractality with increasing degree of disorder. (d): Fractal dimensionality $\mathcal{D}$ as function of the number of random steps $M$. Fractal dimension of the interface grows with increasing randomness and saturates to the value of $\mathcal{D}=1.286 \pm 0.029$ at about $M \simeq 60$. The growing part of $\mathcal{D}(M)$ is well approximated as $\mathcal{D}(M)=c M^{v}+1$ with $c=0.060 \pm 0.01, v=0.335 \pm 0.01$ (the red line). (e): Average width $d$ of the vortex-free shell near the edge of the sample as function of $M$. The red line is $d=c_{1} M^{v_{1}}+c_{2}$ fit with $c_{1}=-0.025 \pm 0.01, c_{2}$ $=0.257 \pm 0.01$, and $v_{1}=0.323 \pm 0.01$.

A special situation, where the defect radius is chosen to be comparable with the superconducting coherence length, $R \gtrsim \xi$ so that $n_{\max }=1$, is identical to a $2 \mathrm{D}$ Coulomb glass of bosons in which the double occupancy is forbidden. The calculated set $\mathbf{d}$ describes thus the distribution of charges corresponding to the minimal energy, i.e. the ground state, of the system. Thus, our finding offers a firm ground for numerical study of hopping conductivity of the Coulomb glass. Using the minimizer $\mathbf{d}$ an initial state in numerical simulations of hopping ensures that the transport indeed occurs due to excitations over the ground state as it should in reality. Note further that although our derivation is done for a particular situation where the lateral size of the system is close to the screening length of the $2 \mathrm{D}$ Coulomb interaction, our results carry importance well beyond their immediate context and break ground for a general analytical description of the ground state of glassy systems.

\section{Methods}

Since the spatial scale of the variation of the order parameter $\xi$ is the smallest scale in the problem we can set the dimensionless order parameter $|u|=1$ everywhere besides the CDs. Then the problem of minimizing GL functional is formulated as a problem of finding a minimizer for the so-called harmonic map-type functional ${ }^{25}$,

$$
F_{\mathrm{G}}[u, \mathbf{A}]=\frac{1}{2} \int_{\Omega}\left[|(\nabla-i \mathbf{A}) u|^{2}+\left(\operatorname{curl} \mathbf{A}-\mathbf{h}_{\mathbf{a}}\right)^{2}\right] d^{2} \mathbf{r} d z
$$

which, in its turn, is reduced to minimizing the energy given by Eq. (2). The solution for the field distribution is sought in a form given by function $\varphi$ of Eq. (4), having the general representation

$$
\varphi(\zeta)=\tilde{\varphi}(\zeta)+\sum_{m=1}^{\mathrm{N}} A_{m} \ln \left(\zeta-a_{m}\right)
$$

where $\tilde{\varphi}(\zeta)$ is single-valued, $A_{\mathrm{m}}$ are real constants ${ }^{23}$, and the summation is taken over all arbitrarily located CDs. By extending this function to the $\mathrm{CD}$ boundaries and taking its derivative $(\psi(\zeta):=d \varphi / d \zeta)$ one arrives at the classical Riemann-Hilbert problem for $\psi$ analytic in the multiply connected domain $D$, which results in a solution in a form of (5). Now the key step is to apply the method of functional equations that allows us to express $\mathbf{d}$ through $\beta_{k}$. Further, it is convenient to minimize numerically the energy as a function in $\beta_{\mathrm{k}}(k=1,2, \ldots, N)$ and after to calculate the optimal values of $\mathbf{d}=\left(d_{1}, \ldots, d_{N}\right)$ by exact formulas through the optimal values of $\beta_{k}$.

1. Binder, K. \& Young, A. P. Spin glasses: experimental facts, theoretical concepts, and open questions. Rev. Mod. Phys. 58, 801-976 (1986).

2. Diep, H. T. Frustrated Spin Systems (World Scientific, 2005).

3. Moessner, R. \& Ramirez, A. P. Geometrical frustration. Phys. Today 59, 24-29 (2006).

4. Pollak, M. Ortuño, M. \& Frydman, A. The Electron Glass. Cambridge University Press, Cambridge (2013).

5. Butko, V. Yu. \& Adams, P. W. Quantum metallicity in a two-dimensional insulator. Nature 409, 161-164 (2001).

6. Sacépé, B. et al. Localization of preformed Cooper pairs in disordered superconductor. Nature Phys. 7, 239244 (2011). 
7. Bouadim, K., Loh, Y. L., Randeria, M. \& Trivedi, N. Single- and two-particle energy gaps across the disorder-driven superconductor-insulator transition. Nature Phys. 7, 884889 (2011).

8. Fisher, M. P. A. Vortex Glass Superconductivity: A Possible New Phase in Bulk High- $T_{c}$ Oxides. Phys. Rev. Lett. 62, 1415-1418 (1989).

9. Nelson, D. R. \& Vinokur, V. M. Boson localization and correlated pinning of superconducting vortex arrays. Phys. Rev. B 48, 13060-13097 (1993).

10. Blatter, J., Feigelman, M. V., Geshkenbein, V. B., Larkin, A. I. \& Vinokur, V. M. Vortices in high temperature superconductors. Rev. Mod. Phys. 66, 1125 (1994).

11. Nattermann, T. \& Scheidl, S. Vortex-glass phases in type-II superconductors. Advances in Physics 49, 607704 (2000).

12. Wasserman, S. \& Faus, K. Social Network Analysis: Methods and Applications 98116 (Cambridge Univ. Press, 1994).

13. Dorogovtsev, S. N., Goltsev, A. V. \& Mendes, J. F. F. Critical phenomena in complex networks. Rev. Mod. Phys. 80, 12751335 (2008).

14. Bryngelson, J. D. \& Wolynes, P. G. Spin glasses and the statistical mechanics of protein folding. Proc. Natl Acad. Sci. USA, 84, 75247528 (1987).

15. Bardalen, E., Bergli, J. \& Galperin, Y. M. Coulomb glasses: A comparison between mean field and Monte Carlo results. Phys. Rev. B85, 155206 (2012).

16. Amir, A., Oreg, Y. \& Imry, Y. Mean-field model for electron-glass dynamics. Phys. Rev. B 77, 165207 (2008).

17. Rammal, R., Tolouse, G. \& Virasoso, M. A. Ultrametricity for physicists, Rev. Mod. Phys. 58 765-788 (1986).

18. Kohsaka, Y., Taylor, C., Fujita, K., Schmidt, A., Lupien, C., Hanaguri, C., Azuma, M., Takano, M., Eisaki, H., Takagi, H., Uchida, S. \& Davis, J. S. An Intrinsic BondCentered Electronic Glass with Unidirectional Domains in Underdoped Cuprates. Science, 315, 1380-1385 (2007).

19. Mkrtchyan, G. S. \& Shmidt, V. V. Interaction Between a Cavity and a Vortex in a Superconductor of the Second Kind. Sov. Phys. JETP 34, 195196 (1972).

20. Berlyand, L. \& Mityushev, V. Generalized Clausius-Mosotti formula for random composite with circular fibers. Journal of Statistical Physics 102 pp. 115-145 (2001).

21. Berlyand, L. \& Mityushev, V. Increase and decrease of the effective conductivity of a two-phase composite due to polydispersity. J. Stat Phys. 118, 3-4 pp. 481-509 (2005).

22. Mityushev, V. Scalar Riemann-Hilbert problem for multiply connected domains. Functional Equations in Mathematical Analysis Springer, ed. Rassias, T. M. \& Brzdek, J. (2012).

23. Mityushev, V. \& Rogosin, S. V. Constructive Methods for Linear and Nonlinear Boundary Value Problems for Analytic Functions. Chapman and Hall/CRC (1999).

24. Mityushev, V. Riemann-Hilbert problems for multiply connected domains and circular slit maps. Computational Methods and Function Theory 11, 575-590 (2011).
25. Iaroshenko, O., Rybalko, V., Vinokur, V. M. \& Berlyand, L. Vortex separation in mesoscopic superconductors. Scientific Reports 3, 1758 (2013).

26. Berlyand, L. \& Rybalko, V. Homogenized description of multiple GinzburgLandau vortices pinned by small holes. special issue NHM 8, 1 pp. 115-130 (2013).

27. Colton, D. \& Kress, R. Integral Equation Methods in Scattering Theory. John Wiley \& Sons (1983).

28. Dassios, G. \& Kleinman, R. Low Frequency Scattering. Oxford Mathematical Monographs (2000).

29. Martin, P. Multiple Scattering: Interaction of time-harmonic waves with $N$ obstacles. Cambridge University Press pp. 284-285 (2006).

30. Barnsley, M. F. \& Rising, H. Fractals everywhere. Elsevier (1993).

\section{Acknowledgments}

The authors would like to thank W. Nawalaniec for the algorithm used to study the transition from periodic to random hole locations in the numerical simulations. Work of L.B. was supported by the NSF grants DMS-1106666 and DMS-1405769, work of V.V. was supported by the U.S. Department of Energy, Office of Science, Materials Sciences and Engineering Division, and work of S.R. and V.M. was partially supported by the NSF grant DMS-1106666.

\section{Author contributions}

V.V. and L.B. conceived and defined the problem, S.R., V.M. and L.B. carried out the calculations. V.V. prepared the manuscript and all the authors contributed to writing, L.B. supervised the project.

\section{Additional information}

Supplementary information accompanies this paper at http://www.nature.com/ scientificreports

Competing financial interests: The authors declare no competing financial interests. How to cite this article: Ryan, S.D., Mityushev, V., Vinokur, V.M. \& Berlyand, L. Rayleigh approximation to ground state of the Bose and Coulomb glasses. Sci. Rep. 5, 7821; DOI:10.1038/srep07821 (2015).

This work is licensed under a Creative Commons Attribution-NonCommercialNoDerivs 4.0 International License. The images or other third party material in this article are included in the article's Creative Commons license, unless indicated otherwise in the credit line; if the material is not included under the Creative Commons license, users will need to obtain permission from the license holder in order to reproduce the material. To view a copy of this license, visit http:// creativecommons.org/licenses/by-nc-nd/4.0/ 